\title{
Zur 4. Jahrestagung der Deutschen Gesellschaft für Afrikanisches Recht.
}

Die 4. Jahrestagung der Deutschen Gesellschaft für Afrikanisches Recht fand am 10. und 11. November 1978 in Heidelberg statt. Der erste Verhandlungstag war dem afrikanischen Genossenschaftsrecht gewidmet und damit einem Gegenstand, dem wegen seiner immensen praktischen Bedeutung besonderes Interesse sicher sein kann, dem rechtsvergleichend gerecht zu werden aber große methodische Schwierigkeiten mit sich bringt. Der auf das Formale beschränkte Organisationsvergleich, der nicht die spezifischen ökonomischen und politischen Funktionen von Genossenschaften in wenig entwickelten Gesellschaften reflektiert, ist unergiebig. Die Tagung vermittelte einen lebhaften Eindruck von den Schwierigkeiten, aber auch dem Reiz der Aufgabe, mit juristischen Mitteln die Rahmenbedingungen für die Entwicklung moderner kooperativer Landwirtschaft zu formulieren und durchzusetzen. Die Diskussion orientierte sich dabei an Vorträgen zweier Studierender des Marburger Instituts für Genossenschaftswesen.

Lipep (Kamerun) berichtete über traditionelle Formen der Zusammenarbeit im afrikanischen Dorfverband, die er mit modernen genossenschaftlichen Strukturen verglich. In den Mittelpunkt seiner Ausführungen stellte er autochtone kamerunische Kooperationsformen in ihrer urspünglichen, noch von europäischen Modellen unbeeinflußten Form. Der Vortrag arbeitete zunächst die wichtigsten Unterschiede zur modernen Genossenschaft heraus, etwa hinsichtlich der Art und Weise des Eintritts oder Ausschlusses (die bei der traditionellen Kooperation meist schwerer wiegende soziale Folgen für die Betroffenen haben als bei der Genossenschaft, auch weniger formalisiert geregelt sind), der Motivation der Organisierung (hier in erster Linie die Notvorsorge, dort das Profitinteresse), der Mitgliedsschaftsrechte und -pflichten (ausdifferenzierte Hierarchien mit zahlreichen Subgruppen, oft auch Diskriminierung einerseits, prinzipielle Gleich heit andererseits) sowie der Ergebnisverteilung (anders als die Genossenschaft kennt die traditionelle Zusammenarbeit in Kamerun kein geregeltes Verhältnis von Leistung und Gegenleistung; es bestehen keine Ansprüche im engeren Sinne, die Gemeinschaft ist auf Bedarfsdeckung hin orientiert). Anders als bei der ausschließlich wirtschaftlichen Zwecken dienenden Genossenschaft will die Kooperation nach traditionellem Muster in erster Linie soziale Ziele verwirklichen, praktiziert Solidarität; die wirtschaftlichen Wirkungen sind in diesen Zusammenhang eingebettet, gleichsam nur Nebenfolgen. Lipep machte jedoch auch verbindende Elemente deutlich und stellte dann die Frage nach den Möglichkeiten und Auswirkungen einer Verschmelzung beider Formen, für die es in Kamerun und anderen afrikanischen Staaten Beispiele gibt. Er neigte dazu, die Aufnahme genossenschaftlicher Elemente in traditionelle Organisationsformen für sinnvoll zu halten, wobei die Entscheidung für oder gegen die Beibehaltung eines traditionellen Strukturelements immer die Auswirkungen auf die soziale Gesamtfunktion der Kooperation im Blick behalten müsse.

Rungapen (Mauritius) gab anschließend einen Úberblick über die Regelung vorgenossenschaftlicher Organisationsformen in afrikanischen Rechtssystemen, also der Phase zwischen dem Entschluß zur Gründung und der Eintragung ins Genossenschaftsregister. Die gesetzliche Regelung dieser Phase, die oft eine , Lernphase“ ist und deshalb einen längeren Zeitraum in Anspruch nimmt, schafft Rechtssicherheit und vermindert das Risiko der Genosssen, die sich erst in die Zusammenarbeit finden müssen. Das Problem der Vorgenossenschaft hat daher in Afrika eine ganz andere praktische Relevanz als etwa in Europa.

Diese Gesichtspunkte - Problem des Aufeinandertreffens an europäischen Vorbildern ausgerichteter Normen mit anders gearteten sozialen Strukturen - beherrschte auch die Diskussion über die beiden Vorträge. Während dabei z. T. die ,,Verwässerung“" genossenschaftli- 
cher Strukturen durch die Aufnahme traditioneller Organisationselemente befürchtet wurde, machten insbesondere die anwesenden afrikanischen Wissenschaftler deutlich, wie die pauschale Úbertragung europäischer Modelle einerseits funktionierende Sozialgefüge zerstören, andererseits aber auch bestehende Unterdrückungs- und Ausbeutungsverhältnisse schlicht bestärken kann: der Häuptling als Genossenschaftsvorsteher. Das letztere Ergebnis kann natürlich auch durch die Verschmelzung traditioneller afrikanischer mit europäischen Modellen bewirkt werden. Die Diskussion zeigte deutlich auf, daß über die Ausgestaltung organisierter Kooperation erst gesprochen werden kann, wenn - länderspezifisch - die grundsätzliche entwicklungspolitische Weiche gestellt ist. -

Die Blickrichtung des zweiten großen Themas der Tagung war eine andere: nicht nach dem Sinn in Europa entstandener Rechtsnormen für afrikanische Verhältnisse wurde gefragt, sondern umgekehrt nach dem Erkenntniswert afrikanischer Normen für das Verständnis der Wirkungsweise von Recht in entwickelten Gesellschaften. Bryde (Hamburg) referierte über „,Die Bedeutung afrikanischer Rechtsethnologie für die Rechtssoziologie“ und beschäftigte sich dabei mit Fragen von Methode und Theorie sowie ,,angewandter" Rechtssoziologie in dem Sinne, daß der Nutzen ethnologischer und soziologischer Forschung für die Rechtspolitik ventiliert wurde. An Beispielen verdeutlichte der Referent, wie Vorgehensweisen, die die ethnologische Dritte-Welt-Forschung entwickelt hat - etwa ihre Bemühung, das Funktionieren einer Gesellschaftsordnung aus sich selbst heraus zu erklären und sie nicht über den Leisten ,,fremder", nur scheinbar allgemeingültiger Kategorien zu schlagen (Stichwort: ,,Immanenz" statt „,Ethnozentrik“) - der rechtssoziologischen Befassung mit Institutionen moderner Industriestaaten nützen können. Der Wert der Erforschung afrikanischen Rechts für allgemeine rechtssoziologische Theoriebildung wurde in der Auseinandersetzung mit Thesen sichtbar, die die Modernität des gesellschaftlichen Phänomens ,,Konflikt“ postulieren: Brydes Ergebnisse aus der Untersuchung afrikanischer Streitschlichtungsmechanismen lassen sie jedenfalls als These mit , ,universalem" Geltungsanspruch als unhaltbar erscheinen. Im Mittelpunkt der Ausführungen zum Wert auf Afrika bezogener Rechtsethnologie und -soziologie für aktuelle Reformüberlegungen zum Recht entwickelter Staaten sowie auch der sich anschließenden Diskussionen stand die Frage nach der Geeignetheit sog. multiplexer Konfliktlösungsmechanismen in entwickelten Gesellschaften: Sind Institutionen, die etwa eine vermögensrechtliche Streitigkeit nicht nur im Hinblick auf das Schicksal einer Geldforderung entscheiden, sondern statt dessen - ,,multiplex” - das soziale Umfeld des konkreten Streits einbeziehen, seine Ursachen angehen, seine Folge- und Begleitererscheinungen regelnd miterfassen, in entwickelten Gesellschaften sinnvoll oder überhaupt nur denkbar? Angesichts der Strukturunterschiede zwischen einer dörflichen Gemeinschaft und etwa der Bevölkerung im Einzugsbereich eines deutschen Amtsgerichts waren sich Referent und Auditorium einig, daß über eine Verpflanzung von Institutionen nicht gesprochen zu werden braucht. Daß aber die Analyse multiplexer Rechtssysteme nicht nur Anlaß zu grundsätzlichen rechtstheoretischen Úberlegungen geben sollte, sondern Úberlegungen über eine der Vielfalt sozialer Beziehungen zwischen Individuen besser gerecht werdende Justiz unmittelbar befruchten kann, wurde durch Brydes Referat nachgewiesen. Die deutsche Gesellschaft für Afrikanisches Recht hat bei der Auswahl der interessanten und anregenden Themen eine glückliche Hand bewiesen: Relevanz des Rechts für afrikanische Entwicklung, Nutzen der Beschäftigung mit afrikanischem Recht für europäische Rechtswissenschaft wurden veranschaulicht. Für künftige Tagungen wäre zu wünschen, daß auch einmal die bislang in Deutschland zu wenig ausgeleuchtete Rolle des Rechts in afrikanischen Diktaturen, insbesondere den letzten Bastionen des Kolonialismus im südlichen Afrika zum Diskussionsgegenstand gemacht wird. Daß im Aufgreifen auch politisch prekärer Themen 
eine genuine Aufgabe wissenschaftlicher Vereinigungen liegt, die hier von anderen Institutionen gelassene Lücken schließen helfen können, sollte außer Frage stehen: Rechtswissenschaft, die Unterprivilegierten nützen kann, hat bei finanziell abhängigen Institutionen keine hinreichende Lobby.

Philip Kunig 